\title{
A graph-theoretic method to study the existence of periodic solutions for a coupled Rayleigh system via inequality techniques
}

\section{Zheng Zhou ${ }^{1 *} \mathbb{D}$, Huaying Liao ${ }^{2}$ and Zhengqiu Zhang ${ }^{3}$}

"Correspondence:

zhouzhengslx@163.com

'School of Applied Mathematical

Science, Xiamen University of

Technology, Xiamen, China

Full list of author information is

available at the end of the article

\begin{abstract}
In the paper, we are concerned with the existence of periodic solutions for a coupled Rayleigh system. By combining graph theory with coincidence degree theory as well as Lyapunov function method, two new sufficient conditions on the existence of periodic solutions for the coupled Rayleigh system are established. Our results on the existence of periodic solutions for the coupled Rayleigh system improve those obtained in the existing literature for coupled Rayleigh system. Hence, our results are new and complementary to the existing papers.
\end{abstract}

First part title: Introduction

Second part title: Preliminaries

Third part title: The existence of periodic solutions

Fourth part title: Numerical test

Five part title: Conclusion

Keywords: Periodic solutions; Coupled Rayleigh system; Graph theory; Continuation theorem of coincidence degree theory; Lyapunov function method

\section{Introduction}

An important class of Rayleigh systems is described by the following form:

$$
x^{\prime \prime}(t)+f\left(t, x^{\prime}(t)\right)+g(t, x(t))=e(t),
$$

where $f, g: R \times R \rightarrow R$ and $e: R \rightarrow R$ are continuous functions. The dynamic behaviors of system (1.1) have been an active research topic due to its extensive applications in physics, mechanics, engineering technique, and other areas (see [1-4] and the references therein). Such successful applications are greatly dependent on the existence of periodic solutions for system (1.1). Hence, the periodicity analysis of system (1.1) has been a subject of intense activities, and many results have been obtained, for example, see [5-8] and the references therein.

In [7], the authors investigated the following Rayleigh type equation:

$$
x^{\prime \prime}(t)+f\left(x^{\prime}(t)\right)+g(t, x(t))=e(t)
$$

(c) The Author(s) 2019. This article is distributed under the terms of the Creative Commons Attribution 4.0 International License (http://creativecommons.org/licenses/by/4.0/), which permits unrestricted use, distribution, and reproduction in any medium, provided you give appropriate credit to the original author(s) and the source, provide a link to the Creative Commons license, and indicate if changes were made. 
where $f: R \rightarrow R$ is continuous, $g: R^{2} \rightarrow R$ is continuous and $T$-periodic with respect to the first variable. Some criteria to guarantee the existence of periodic solutions of this equation were presented in [7] by using Mawhin's continuation theorem, Floquet theory, Lyapunov stability theory, and some analysis techniques. In [6], the authors studied the existence of periodic solutions of Rayleigh equations:

$$
x^{\prime \prime}(t)+f\left(t, x^{\prime}\right)+g(x)=e(t),
$$

where $f: R^{2} \rightarrow R$ is continuous and $T$-periodic with respect to the first variable, $g, e: R \rightarrow$ $R$ are continuous, and $e$ is $T$-periodic. They proved that the given equation possesses at least one $T$-periodic solution under some conditions. In [5], by employing the continuation theorem of coincidence degree theory, the authors studied a kind of Rayleigh equation with a deviating argument as follows:

$$
x^{\prime \prime}(t)+f\left(x^{\prime}(t)\right)+g(x(t-\tau(t)))=p(t)
$$

where $g, f: R \rightarrow R$ are two continuous functions, $\tau(t)$ and $p(t)$ are continuous and $T$ periodic functions, and established some new results on the existence of periodic solutions for system (1.4).

With the popularity of coupled systems, so far, the existence and global stability of periodic solutions of coupled systems on neural networks have gained increasing research [9-13], the existence of periodic solutions of coupled systems on the predator-prey systems $[14,15]$ has been widely studied, the existence of periodic solutions and stability of equilibrium point for coupled systems on networks have been widely investigated, for example, see [16-23] and the references therein.

In [21], the authors were concerned with the following coupled Rayleigh system:

$$
x_{k}^{\prime \prime}(t)+f_{k}\left(t, x_{k}^{\prime}(t)\right)+g_{k}\left(t, x_{k}(t)\right)=e_{k}(t),
$$

where $k=1,2, \ldots, n, n$ is a positive integer, $f_{k}, g_{k}: R \rightarrow R$ and $e_{k}: R \rightarrow R$ are continuous $\omega$-periodic functions in the first argument with period $\omega>0, f_{k}\left(t, x_{k}\right)$ is continuously differentiable in $x_{k}$.

In [21], by taking $y_{k}(t)=x_{k}^{\prime}(t)+\eta x_{k}(t), \eta>0$, system (1.5) was rewritten as

$$
\left\{\begin{array}{l}
x_{k}^{\prime}(t)=y_{k}(t)-\eta x_{k}(t), \\
y_{k}^{\prime}(t)=-\eta^{2} x_{k}(t)+\eta y_{k}(t)-f_{k}\left(t, y_{k}(t)-\eta x_{k}(t)\right)-g_{k}\left(t, x_{k}(t)\right)+e(t) .
\end{array}\right.
$$

By adding $-\sum_{h=1}^{l} a_{k h}\left(y_{k}(t)-y_{h}(t)\right)$ into the second equation of system (1.6), in [21], the authors established the following linear coupled Rayleigh system:

$$
\left\{\begin{aligned}
x_{k}^{\prime}(t)= & y_{k}(t)-\eta x_{k}(t), \\
y_{k}^{\prime}(t)= & -\eta^{2} x_{k}(t)+\eta y_{k}(t)-f_{k}\left(t, y_{k}(t)-\eta x_{k}(t)\right)-g_{k}\left(t, x_{k}(t)\right)+e(t) \\
& -\sum_{h=1}^{l} a_{k h}\left(y_{k}(t)-y_{h}(t)\right), \quad k \in K,
\end{aligned}\right.
$$

where $a_{k h}\left(y_{h}-y_{k}\right)$ represents the influence of vertex $h$ on vertex $k, a_{k h}>0$, and $a_{k h}=0$ if and only if there exists no arc from vertex $h$ to vertex $k$ in $g, K, g$ are defined in Definition 2.1. 
In [21, 24-26], by combining graph theory with coincidence degree theory as well as Lyapunov method, a sufficient criterion for the existence of periodic solutions for system (1.7) was provided under these conditions $\left(A_{1}\right)-\left(A_{5}\right)$.

However, the conditions in the results obtained in [21] on the existence of periodic solutions for the coupled system (1.7) are too complicated and there are too many of them. This motivates us to obtain more concise and easily verified new sufficient conditions for system (1.7).

Up to now, the global existence of periodic solutions for differential systems has been investigated mainly by employing the following five methods: (1) Fixed point theorem methods [26]; (2) Combining continuation theorem of coincidence degree theory with the a priori estimate of periodic solutions [9, 11, 13-15, 27-30]; (3) Combining continuation theorem of coincidence degree theory with LMI [12]; (4) Combining continuation theorem of coincidence degree theory with Lyapunov function method [16, 18-21, 31]; (5) The method of upper and lower functions. But, in the above-mentioned methods, (3) and (4) are used in recent years to study the existence of periodic solutions for different systems. In this paper, we apply (4) to study the existence of periodic solution for system (1.7), but the concrete analysis techniques in our paper are different from those used in $[16,18-21]$. In this paper, our purpose is, by combining graph theory with Mawhin's continuation theorem of coincidence degree theory as well as Lyapunov functional method, to improve the results on the existence of periodic solutions obtained in [21] for system (1.7) by removing conditions $\left(A_{4}\right)$ and $\left(A_{5}\right)$ in [21]. Consequently, the contribution of this paper lies in the following two aspects: (1) Novel inequality techniques are cited to study the existence of periodic solutions for different equations; (2) Novel sufficient conditions are gained for system (1.7) by improving the results obtained in the existing papers.

This paper is organized as follows. Some preliminaries and lemmas are given in Sect. 2. In Sect. 3, two sufficient conditions are derived for the existence of periodic solutions for system (1.7). In Sect. 4, two illustrative examples are given to show the effectiveness of the proposed theory. In Sect. 5, a conclusion is given.

\section{Preliminaries}

Let $R$ and $R^{n}$ be the set of real numbers and an $\mathrm{n}$-dimensional Euclidean space, respectively. Let $|\cdot|$ and $\|\cdot\|$ respectively be norms of $R$ and $R^{n}$.

We cite the notation as follows:

$$
\bar{f}=\max _{t \in[0, \omega]}\{|f(t)|\}
$$

where $f(t)$ is a continuous $\omega$-periodic function.

We make the assumptions as follows:

$\left(H_{1}\right)$ There exist constants $b>0, d>0$ such that, for $k \in K$,

$$
\left|g_{k}\left(t, x_{k}\right)\right| \leq b\left|x_{k}(t)\right|+d
$$

$\left(H_{2}\right)$ There exist constants $\delta<0, r>0, e>0$, and $a$ with $A=-\eta-\delta \eta^{2}+0.5 b^{2}+0.5 b d+$ $\eta^{2}+0.5 \eta r+0.5 \eta|a|+0.5 \eta e<0$ such that, for $k \in K$,

$$
x_{k} f_{k}\left(t, x_{k}\right) \geq \delta x_{k}^{2}+a x_{k},\left|f_{k}\left(t, x_{k}\right)\right| \leq r\left|x_{k}\right|+e .
$$


$\left(A_{1}\right)$ There exist constants $\delta$ and $\mu_{1}$ satisfying $2 \delta-\mu_{1} \geq 1-\frac{\eta^{2}}{2}$ such that, for $k \in K$,

$$
x_{k} g_{k}\left(t, x_{k}\right) \geq \delta x_{k}^{2}, g_{k}^{2}\left(t, x_{k}\right) \leq \mu_{1} x_{k}^{2}
$$

$\left(A_{2}\right)$ Function $f_{k}\left(t, x_{k}\right)$ satisfies, for $k \in K$,

$$
0<\frac{2(\eta+1)}{2-\eta} \leq \frac{f_{k}\left(t, x_{k}\right)}{x_{k}} \leq 2, x_{k} \neq 0 .
$$

$\left(A_{3}\right)$ The digraph $(g, B)\left(B=\left(a_{k h}\right)_{n \times l}\right)$ is strongly connected.

$\left(A_{4}\right)$ There exists $\varepsilon>0$ such that, for $k \in K$,

$$
m_{k}\left(x_{k}\right)=\frac{\frac{1}{\omega} \int_{0}^{\omega} g_{k}\left(t, x_{k}\right) d t}{x_{k}} \geq \varepsilon, \quad x_{k} \neq 0,
$$

where $m_{k}\left(x_{k}\right) \in C^{1}(R, R)$.

$\left(A_{5}\right)$ For $k \in K$

$$
\int_{0}^{\omega} e_{k}(t) d t=0
$$

For the sake of convenience, we introduce Gaines and Mawhin's continuation theorem about coincidence degree theory [24] and graph theory [25] as follows.

Lemma 2.1 ([24]) Assume that $X$ and $Z$ are two Banach spaces, $L: D(L) \subset X \rightarrow Z$ is a Fredholm operator with index zero. Let $\Omega \in X$ be an open bounded set and $N: \bar{\Omega} \rightarrow Z$ be L-compact on $\bar{\Omega}$. Assume that

(1) for each $\lambda \in(0,1), u \in \partial \Omega \cap \operatorname{Dom} L, L u \neq \lambda N u$;

(2) for each $u \in \partial \Omega \cap \operatorname{Ker} L, Q N u \neq 0$;

(3) $\operatorname{deg}\{J Q N u, \Omega \cap \operatorname{Ker} L, 0\} \neq 0$, where $\operatorname{deg}$ denotes the Brouwer degree.

Then the operator equation $L u=N u$ has at least one solution in $\bar{\Omega} \cap \operatorname{Dom} L$.

Definition 2.1 ([23]) A directed graph $g=(U, K)$ contains a set $U=\{1,2, \ldots, n\}$ of vertices and a set $K$ of $\operatorname{arcs}(i, j)$ leading from initial vertex $i$ to terminal vertex $j$. A subgraph $\Gamma$ of $g$ is said to be spanning if $\Gamma$ and $g$ have the same vertex set. A directed graph $g$ is weighed if each arc $(j, i)$ is assigned a positive weight $b_{i j}$. The weight $W(\Gamma)$ of a subgraph $H$ is the product of the weights on all its arc. A directed path $\delta$ in $g$ is subgraph with distinct vertices $\left\{i_{1}, i_{2}, \ldots, i_{m}\right\}$ such that its set of arcs is $\left\{\left(i_{k}, i_{k+1}\right): k=1,2, \ldots, m-1\right\}$. For a weighted digraph $g$ with $l$ vertices, we define the weight matrix $B=\left(b_{i j}\right)_{n \times n}$ whose entry $b_{i j}>0$ is equal to the weight of $\operatorname{arc}(j, i)$ if it exists, and 0 otherwise. A digraph $g$ is strongly connected if, for any pair of distinct vertices, there exists a directed path from one to the other. The Laplacian matrix of $(g, B)$ is defined as $L=\left(p_{i j}\right)_{l \times l}$, where $p_{i j}=-b_{i j}$ for $i \neq j$ and $p_{i j}=\sum_{k \neq i} b_{i k}$ for $i=j$.

Lemma 2.2 ([24]) Suppose that $l \geq 2$ and $c_{k}$ denotes the cofactor of the kth diagonal element of the Laplacian matrix of $(g, B)$. Then $\sum_{k, h=1}^{l} c_{k} a_{k h} G_{k h}\left(x_{k}, x_{h}\right)=\sum_{Q \in \Omega} W(Q) \times$ $\sum_{(k, h) \in K\left(C_{Q}\right)} G_{h k}\left(x_{h}, x_{k}\right)$, where $G_{k h}\left(x_{k}, x_{h}\right)$ is an arbitrary function, $Q$ is the set of all spanning unicyclic graphs of $(g, B), W(Q)$ is the weight of $Q, C_{Q}$ denotes the directed cycle of $Q$, and $K\left(C_{Q}\right)$ is the set of arcs in $C_{Q}$. In particular, if $(g, B)$ is strongly connected, then $c_{k}>0$ for $1 \leq k \leq l$. 
Lemma 2.3 For any $\lambda \in(0,1)$, consider the following system:

$$
\left\{\begin{aligned}
x_{k}^{\prime}(t)= & \lambda\left[y_{k}(t)-\eta x_{k}(t)\right], \\
y_{k}^{\prime}(t)= & \lambda\left[-\eta^{2} x_{k}(t)+\eta y_{k}(t)-f_{k}\left(t, y_{k}(t)-\eta x_{k}(t)\right)-g_{k}\left(t, x_{k}(t)\right)+e_{k}(t)\right. \\
& \left.-\sum_{h=1}^{l} a_{k h}\left(y_{k}(t)-y_{h}(t)\right)\right], \quad k \in K .
\end{aligned}\right.
$$

If the periodic solutions of system (2.1) exist, then they are bounded and the boundary is independent of the choice of $\lambda$ under assumptions $\left(H_{1}\right),\left(H_{2}\right)$, and $\left(A_{3}\right)$. Namely, there exists a positive constant $H$ such that

$$
\left\|(x(t), y(t))^{T}\right\|=\left\|\left(x_{1}(t), x_{2}(t), \ldots, x_{l}(t), y_{1}(t), y_{2}(t), \ldots, y_{l}(t)\right)^{T}\right\| \leq H
$$

the norm $\|\cdot\|$ is defined in the proof of Theorem 3.1 .

Proof Suppose that $(x(t), y(t))^{T}=\left(x_{1}(t), x_{2}(t), \ldots, x_{l}(t), y_{1}(t), y_{2}(t), \ldots, y_{l}(t)\right)^{T}$ is a periodic solution of system (2.1) for some $\lambda \in(0,1)$. Letting $V(x, y)=0.5 \sum_{k=1}^{l} c_{k}\left(x_{k}^{2}+y_{k}^{2}\right)$, where $c_{k}$ denotes the cofactor of the kth diagonal element of Laplacian matrix of $\left(g,\left(b_{k h}\right)_{l \times l}\right)$. According to assumption $\left(A_{3}\right)$ and Lemma 2.2, one has $c_{k}>0, k \in K$. Making use of assumptions $\left(H_{1}\right)$ and $\left(H_{2}\right)$, we have

$$
\begin{aligned}
\frac{d V(x, y)}{d t} & \lambda \sum_{k=1}^{l} c_{k}\left[-\eta x_{k}^{2}(t)-\eta^{2} x_{k}(t) y_{k}(t)+\eta y_{k}^{2}(t)-y_{k}(t) f_{k}\left(t, y_{k}(t)-\eta x_{k}(t)\right)\right. \\
& \left.+y_{k}(t)\left(x_{k}(t)-g_{k}\left(t, x_{k}(t)\right)\right)+y_{k}(t) e_{k}(t)-\sum_{h=1}^{l} a_{k h} y_{k}(t)\left(y_{k}(t)-y_{h}(t)\right)\right] \\
& \\
& \quad \lambda \sum_{k=1}^{l} c_{k}\left\{-\eta x_{k}^{2}(t)-\eta^{2} x_{k}(t) y_{k}(t)+\eta y_{k}^{2}(t)-\delta\left[y_{k}(t)-\eta x_{k}(t)\right]^{2}-a\left[y_{k}(t)-\eta x_{k}(t)\right]\right. \\
& +y_{k}(t) \times e_{k}(t)+x_{k}(t) y_{k}(t)+0.5 y_{k}^{2}(t)+0.5 g_{k}^{2}\left(t, x_{k}\right)-\eta x_{k}(t) f_{k}\left(t, y_{k}(t)-\eta x_{k}(t)\right) \\
& \left.-\sum_{h=1}^{l} a_{k h} y_{k}^{2}(t)+\frac{1}{2} \sum_{h=1}^{l} a_{k h}\left[y_{k}^{2}(t)+y_{h}^{2}(t)\right]\right\} \\
\leq & \lambda \sum_{k=1}^{l} c_{k}\left\{-\eta x_{k}^{2}(t)-\eta^{2} x_{k}(t) y_{k}(t)+\eta y_{k}^{2}(t)-\delta\left[y_{k}(t)-\eta x_{k}(t)\right]^{2}-a\left[y_{k}(t)-\eta x_{k}(t)\right]\right. \\
& +y_{k}(t) e_{k}+0.5 y_{k}^{2}(t)+y_{k}(t) x_{k}(t)+0.5\left[b^{2} x_{k}^{2}(t)+d^{2}+2 b d\left|x_{k}(t)\right|\right] \\
& +\eta\left|x_{k}(t)\right|\left[r\left|y_{k}(t)\right|+r \eta\left|x_{k}(t)\right|+e\right]+\frac{1}{2} \sum_{h=1}^{l} c_{k h}\left\{\left(-\eta y_{h}^{2}(t)-y_{k}^{2}(t)\right]\right\}
\end{aligned}
$$




$$
\begin{aligned}
& +(\eta-\delta+0.5 \eta r+0.5|a|+1) y_{k}^{2}(t)+\left(-\eta^{2}+2 \eta \delta+\right) x_{k}(t) y_{k}(t) \\
& \left.+0.5\left(\left(\overline{e_{k}}\right)^{2}+b d+d^{2}+\eta e+|a| \eta+|a|\right)\right\}+\frac{\lambda}{2} \sum_{h=1, h}^{l} c_{k} a_{k h} F_{h k}\left(y_{k}, y_{h}\right)
\end{aligned}
$$

where $F_{h k}\left(y_{k}, y_{h}\right)=y_{h}^{2}-y_{k}^{2}$. By employing Lemma 2.2, we obtain

$$
\sum_{k, h=1}^{l} c_{k} a_{k h} F_{h k}\left(y_{k}, y_{h}\right)=0
$$

from which, together with (2.2), it follows that

$$
\begin{aligned}
& \frac{d V(x, y)}{d t} \\
& \leq \lambda \sum_{k=1}^{l} c_{k}\left\{\left[-\eta+\left(-\delta \eta^{2}+0.5 b^{2}+0.5 b d+\eta^{2} l+0.5 \eta l+0.5 \eta|a|+0.5 \eta e\right)\right] x_{k}^{2}(t)\right. \\
& \quad+(\eta-\delta+0.5 \eta l+0.5|a|+1) y_{k}^{2}(t)+\left(-\eta^{2}+2 \eta \delta+1\right) x_{k}(t) y_{k}(t) \\
& \left.\quad+0.5\left(\left(\overline{e_{k}}\right)^{2}+b d+d^{2}+\eta e+|a| \eta+|a|\right)\right\} .
\end{aligned}
$$

Since $A<0, \delta<0$, then $\left(-\eta^{2}+2 \eta \delta+1\right)^{2} y_{k}^{2}(t)-4 A(\eta-\delta+0.5 \eta l+0.5|a|+1) y_{k}^{2}(t)>0$, $\forall y_{k}(t) \neq 0, x_{k}(t) \neq 0$. So the equation in $x_{k}(t): A x_{k}^{2}(t)+(\eta-\delta+0.5 \eta l+0.5|a|+1) y_{k}^{2}(t)+$ $\left(-\eta^{2}+2 \eta \delta+1\right) x_{k}(t) y_{k}(t)=0$ has two real roots $x_{1}, x_{2}\left(x_{1}<x_{2}\right)$ for fixed $k$ and

$$
x_{1}=\frac{-B-\sqrt{B^{2}-4 A C}}{2 A} y_{k}(t), \quad x_{2}=\frac{-B+\sqrt{B^{2}-4 A C}}{2 A} y_{k}(t) .
$$

Hence, when $x_{k}>x_{2}$, or $x_{k}<x_{1}, A x_{k}^{2}(t)+(\eta-\delta+0.5 \eta l+0.5|a|+1) y_{k}^{2}(t)+\left(-\eta^{2}+2 \eta \delta+\right.$ 1) $x_{k}(t) y_{k}(t)<0$. Namely, when $\left|x_{k}\right|>\max \left\{\left|x_{1}\right|,\left|x_{2}\right|\right\}=r^{*}\left|y_{k}\right|, A x_{k}^{2}(t)+(\eta-\delta+0.5 \eta l+0.5|a|+$ 1) $y_{k}^{2}(t)+\left(-\eta^{2}+2 \eta \delta+1\right) x_{k}(t) y_{k}(t)<0$. So when

$$
\begin{aligned}
\begin{aligned}
\left\|(x, y)^{T}\right\| & =\left\|\left(x_{1}, x_{2}, \ldots, x_{n}, y_{1}, y_{2}, \ldots, y_{n}\right)^{T}\right\| \\
& =\sum_{k=1}^{n}\left[\max _{t \in[0, \omega]}\left(\left|x_{k}(t)\right|+\left|y_{k}(t)\right|\right)\right]>n\left(r^{*}+1\right)\left|y_{k}\right|,
\end{aligned} \\
A x_{k}^{2}(t)+(\eta-\delta+0.5 \eta r+0.5|a|+1) y_{k}^{2}(t)+\left(-\eta^{2}+2 \eta \delta+1\right) x_{k}(t) y_{k}(t)<0 .
\end{aligned}
$$

So there exists a positive constant $r_{1}$ such that, when $\left\|(x, y)^{T}\right\|>r_{1}$,

$$
A x_{k}^{2}(t)+(\eta-\delta+0.5 \eta r+0.5|a|+1) y_{k}^{2}(t)+\left(-\eta^{2}+2 \eta \delta+1\right) x_{k}(t) y_{k}(t)<0 \text {. }
$$

Since $A x_{k}^{2}(t)+(\eta-\delta+0.5 \eta r+0.5|a|+1) y_{k}^{2}(t)+\left(-\eta^{2}+2 \eta \delta+1\right) x_{k}(t) y_{k}(t)$ is decreasing in $x_{k}$ when $x_{k}>r\left|y_{k}\right|$, and is increasing in $x_{k}$ when $x_{k}<-r^{*}\left|y_{k}\right|$, hence there exists a positive $H$ such that, when $\left\|(x, y)^{T}\right\|>H$,

$$
\begin{aligned}
& A x_{k}^{2}(t)+q_{k}(\eta-\delta+0.5 \eta r+0.5|a|+1) y_{k}^{2}(t)+\left(-\eta^{2}+2 \eta \delta+1\right) x_{k}(t) y_{k}(t) \\
& \quad+0.5\left(\left(\overline{e_{k}}\right)^{2}+b d+d^{2}+\eta e+|a| \eta+|a|\right)<0 .
\end{aligned}
$$


From (2.3), it follows that there exists a positive constant $H$, which is independent of $\lambda$, such that when

$$
\left\|(x, y)^{T}\right\| \geq H, \quad \frac{d V(x, y)}{d t} \leq 0 .
$$

Recalling the fact that $(x(t), y(t))^{T}$ is an $\omega$-periodic solution, we see that $V(x(t), y(t))$ is an $\omega$-periodic solution. On the other hand, if $\left\|(x(t), y(t))^{T}\right\| \geq H$, then $\frac{d V(x(t), y(t))}{d t} \leq 0$, which is in contradiction to the fact $V(x(t), y(t))$ is a continuous $\omega$-periodic solution. Thus $\left\|(x(t), y(t))^{T}\right\| \leq H$.

Remark 1 From the proof of Lemma 2.3, there exists a positive $H$ such that when $\left\|(x, y)^{T}\right\|>H$, then

$$
\begin{aligned}
& A x_{k}^{2}(t)+q_{k}(\eta-\delta+0.5 \eta r+0.5|a|+1) y_{k}^{2}(t)+\left(-\eta^{2}+2 \eta \delta+1\right) x_{k}(t) y_{k}(t) \\
& \quad+0.5\left(\left(\overline{e_{k}}\right)^{2}+b d+d^{2}+\eta e+|a| \eta+|a|\right)<0 .
\end{aligned}
$$

\section{The existence of periodic solutions}

In this section, we establish two sufficient conditions on the existence of periodic solutions for system (1.7) by combining graph theory with Mawhin's continuation theorem of coincidence degree theory.

Theorem 3.1 Under assumptions $\left(H_{1}\right),\left(H_{2}\right)$, and assumption $\left(A_{3}\right)$, system $(1.7)$ has at least an $\omega$-periodic solution.

Proof We will establish the existence of periodic solutions of system (1.7) by using Lemma 2.1. Let

$$
\begin{aligned}
X=Z= & \left\{z=(x(t), y(t))^{T}=\left(x_{1}(t), x_{2}(t), \ldots, x_{l}(t), y_{1}(t), y_{2}(t), \ldots, y_{l}(t)\right)^{T}:\right. \\
& (x(t), y(t))^{T} \in C^{1}\left(R, R^{2 l}\right), x_{i}(t+\omega)=x_{i}(t), y_{i}(t+\omega)=y_{i}(t) \\
& (i=1,2, \ldots, l), t \in R\} .
\end{aligned}
$$

Denote

$$
\left\|(x(t), y(t))^{T}\right\|=\sum_{k=1}^{l}\left[\max _{t \in[0, \omega]}\left|x_{k}(t)\right|+\max _{t \in[0, \omega]}\left|y_{k}(t)\right|\right] .
$$

Then $X$ and $Z$ are Banach spaces with the norm $\|\cdot\|$. Set

$$
\begin{aligned}
G_{k}=y_{k}(t)-\eta x_{k}(t), \\
\begin{aligned}
F_{k}(t)= & -\eta^{2} x_{k}(t)+\eta y_{k}(t)-f_{k}\left(t, y_{k}(t)-\eta x_{k}(t)\right)-g_{k}\left(t, x_{k}(t)\right)+e_{k}(t) \\
& -\sum_{h=1}^{l} a_{k h}\left(y_{k}(t)-y_{h}(t)\right), \quad k=1,2, \ldots, l . \\
L z=z^{\prime}= & \left(x^{\prime}(t), y^{\prime}(t)\right)^{T}=\left(x_{1}^{\prime}(t), x_{2}^{\prime}(t), \ldots, x_{l}^{\prime}(t), y_{1}^{\prime}(t), \ldots, y_{l}^{\prime}(t)\right)^{T},
\end{aligned}
\end{aligned}
$$




$$
\begin{aligned}
& N z=\left(G_{1}(t), G_{2}(t), \ldots, G_{l}(t), F_{1}(t), F_{2}(t), \ldots, F_{l}(t)\right), \\
& P z=\frac{1}{\omega} \int_{0}^{\omega} z(t) d t, \quad z \in X ; \quad Q z=\frac{1}{\omega} \int_{0}^{\omega} z(t) d t, \quad z \in Z .
\end{aligned}
$$

It is easy to show that $\operatorname{Dim} \operatorname{Ker} L=\operatorname{Dim} R^{2 l}=2 l=\operatorname{codim} \operatorname{Im} L$. Hence, $L$ is a Fredholm mapping of index zero. We can prove that

$$
\operatorname{Im} P=\operatorname{Ker} L, \quad \operatorname{Im} L=\operatorname{Ker} Q=\operatorname{Im}(I-Q) .
$$

Furthermore, the generalized inverse $K_{P}$ of $L$ is as follows: $K_{P}: \operatorname{Im} L \rightarrow \operatorname{Ker} P \cap \operatorname{Dom} L$ exists and

$$
K_{P}(z)=\int_{0}^{t} z(s) d s-\frac{1}{\omega} \int_{0}^{\omega} \int_{0}^{t} z(s) d s d t
$$

Thus

$$
\begin{aligned}
& Q N z \\
& =\left(\frac{1}{\omega} \int_{0}^{\omega} G_{1}(t) d t, \frac{1}{\omega} \int_{0}^{\omega} G_{2}(t) d t, \ldots, \frac{1}{\omega} \int_{0}^{\omega} G_{n}(t) d t,\right. \\
& \\
& \left.\quad \frac{1}{\omega} \int_{0}^{\omega} F_{1}(t) d t, \frac{1}{\omega} \int_{0}^{\omega} F_{2}(t) d t, \frac{1}{\omega} \int_{0}^{\omega} F_{n}(t) d t\right)^{T}
\end{aligned}
$$

and

$$
\begin{aligned}
& K_{P}(I-Q) N z \\
& \quad=\left(\begin{array}{c}
\int_{0}^{t} G_{1}(s) d s-\frac{1}{\omega} \int_{0}^{\omega} \int_{0}^{t} G_{1}(s) d s d t-\left(\frac{t}{\omega}-\frac{1}{2}\right) \int_{0}^{\omega} G_{1}(t) d t \\
\int_{0}^{t} G_{2}(s) d s-\frac{1}{\omega} \int_{0}^{\omega} \int_{0}^{t} G_{2}(s) d s d t-\left(\frac{t}{\omega}-\frac{1}{2}\right) \int_{0}^{\omega} G_{2}(t) d t \\
\ldots \\
\int_{0}^{t} G_{n}(s) d s-\frac{1}{\omega} \int_{0}^{\omega} \int_{0}^{t} G_{n}(s) d s d t-\left(\frac{t}{\omega}-\frac{1}{2}\right) \int_{0}^{\omega} G_{n}(t) d t \\
\int_{0}^{t} F_{1}(s) d s-\frac{1}{\omega} \int_{0}^{\omega} \int_{0}^{t} F_{1}(s) d s d t-\left(\frac{t}{\omega}-\frac{1}{2}\right) \int_{0}^{\omega} F_{1}(t) d t \\
\int_{0}^{t} F_{2}(s) d s-\frac{1}{\omega} \int_{0}^{\omega} \int_{0}^{t} F_{2}(s) d s d t-\left(\frac{t}{\omega}-\frac{1}{2}\right) \int_{0}^{\omega} F_{2}(t) d t \\
\ldots \\
\int_{0}^{t} F_{n}(s) d s-\frac{1}{\omega} \int_{0}^{\omega} \int_{0}^{t} F_{n}(s) d s d t-\left(\frac{t}{\omega}-\frac{1}{2}\right) \int_{0}^{\omega} F_{n}(t) d t
\end{array}\right)^{T}
\end{aligned}
$$

Clearly, $Q N$ and $K_{P}(I-Q) N$ are continuous and $Q N(\bar{\Omega})$ is bounded, where $\Omega$ is an open set in $X$. Then by Arzela-Ascoli theorem, we can prove that $\overline{K_{P}(I-Q) N(\bar{\Omega})}$ is compact. Hence, $N$ is $L$-compact on $\bar{\Omega}$.

Corresponding to the operator equation $L x=\lambda N x, \lambda \in(0,1)$, we have system (2.1). By Lemma 2.3, for every periodic solution $(x(t), y(t))^{T}=\left(x_{1}(t), x_{2}(t), \ldots, x_{l}(t), y_{1}(t), \ldots, y_{l}(t)\right)^{T}$ of $L z=\lambda N z$, there is $H>0$, which is independent of the choice of $\lambda$, such that $\|(x(t)$, $y(t))^{T} \|<H$.

We set $\Omega=\left\{(x(t), y(t))^{T} \in X:\left\|(x, y)^{T}\right\|<H+r\right\}$, where $r>0$ is chosen so that the bound is larger. Hence, for any $\lambda \in(0,1), z \in \partial \Omega \cap \operatorname{Dom} L, L z \neq \lambda N z$. When $z \in \partial \Omega \cap \operatorname{Ker} P$, we will show $Q N z \neq 0$. When $z \in \partial \Omega \cap \operatorname{Ker} L, z \in R^{2 l}$ (namely $z$ is a constant vector) with 
$\|z\|=\left\|(x, y)^{T}\right\|=H+r$. If $z$ is a constant vector with $\|z\|=H+r, Q N z=0$, then it follows that the constant vector $z$ with $\|z\|=H+r$ satisfies, for $k=1,2, \ldots, l$,

$$
\frac{1}{\omega} \int_{0}^{\omega} G_{k}(t) d t=0, \quad \frac{1}{\omega} \int_{0}^{\omega} F_{k}(t) d t=0 .
$$

Hence, there exist $t_{k}(i=1,2), \xi_{i} \in[0, \omega](k=1,2, \ldots, l)$ such that

$$
G_{k}\left(t_{k}\right)=0, \quad F_{k}\left(\xi_{k}\right)=0
$$

From (3.1), we have

$$
0=\sum_{k=1}^{l} c_{k}\left(x_{k} G_{k}\left(t_{k}\right)+y_{k} F_{k}\left(\xi_{k}\right)\right)
$$

By using the same proof as those of (2.4) in Lemma 2.3, from (3.2), it follows that

$$
\begin{aligned}
0= & \sum_{k=1}^{l} c_{k}\left(x_{k} G_{k}\left(t_{k}\right)+y_{k} F_{k}\left(\xi_{k}\right)\right) \\
\leq & \sum_{k=1}^{l} c_{k}\left\{\left[-\eta+\left(-\delta \eta^{2}+0.5 b^{2}+0.5 b d+\eta^{2} r+0.5 \eta l+0.5 \eta|a|+0.5 \eta e\right)\right] x_{k}^{2}\right. \\
& +(\eta-\delta+0.5 \eta r+0.5|a|+1) y_{k}^{2}+\left(-\eta^{2}+2 \eta \delta+1\right) x_{k} y_{k} \\
& \left.+0.5\left(\left(\overline{e_{k}}\right)^{2}+b d+d^{2}+\eta e+|a| \eta+|a|\right)\right\} .
\end{aligned}
$$

It follows from Remark 1 that, since $\left\|(x, y)^{T}\right\|>H$,

$$
\begin{aligned}
& \sum_{k=1}^{l}\left\{\left[-\eta+\left(-\delta \eta^{2}+0.5 b^{2}+0.5 b d+\eta^{2} r+0.5 \eta l+0.5 \eta|a|+0.5 \eta e\right)\right] x_{k}^{2}\right. \\
& \quad+(\eta-\delta+0.5 \eta r+0.5|a|+1) y_{k}^{2}+\left(-\eta^{2}+2 \eta \delta+1\right) x_{k} y_{k} \\
& \left.\quad+0.5\left(\left(\overline{e_{k}}\right)^{2}+b d+d^{2}+\eta+|a| \eta+|a|\right)\right\}<0
\end{aligned}
$$

which contradicts with (3.3). Hence, for each $z \in \partial \cap \operatorname{Ker} L, Q N z \neq 0$.

Finally, we show that $\operatorname{deg}_{B}\{J Q N, \Omega \cap \operatorname{Ker} L,(0,0, \ldots, 0)\} \neq 0$. We only show that $\operatorname{deg}_{B}\{J Q N z, \Omega \cap \operatorname{Ker} L,(0,0, \ldots, 0)\} \neq 0$, when $z \in \partial \Omega \cap \operatorname{Ker} L$. To this end, we construct the following mapping for $k=1,2, \ldots, l$ :

$$
\begin{aligned}
L(x, y, \mu) & \\
= & (1-\mu)\left(y_{1}-\eta x_{1}, y_{2}-\eta x_{2}, \ldots, y_{n}-\eta x_{n},\right. \\
& -\eta^{2} x_{1}+\eta y_{1}-f_{1}\left(\xi_{1}, y_{1}-\eta x_{1}\right)-g_{1}\left(\xi_{1}, x_{1}\right)+e_{1}\left(\xi_{1}\right)-\sum_{h=1}^{l} a_{1 h}\left(y_{1}-y_{h}\right), \\
& -\eta^{2} x_{2}+\eta y_{2}-f_{2}\left(\xi_{2}, y_{2}-\eta x_{2}\right)-g_{2}\left(\xi_{2}, x_{2}\right)+e_{2}\left(\xi_{2}\right)-\sum_{h=1}^{l} a_{2 h} \times\left(y_{2}-y_{h}\right),
\end{aligned}
$$




$$
\begin{aligned}
& \cdots \\
& \left.-\eta^{2} x_{l}+\eta y_{l}-f_{l}\left(\xi_{l}, y_{l}-\eta x_{l}\right)-g_{l}\left(\xi_{l}, x_{l}\right)+e_{l}\left(\xi_{l}\right)-\sum_{h=1}^{l} a_{l h}\left(y_{l}-y_{h}\right)\right) \\
& +\mu\left(m_{1} x_{1}+n_{1} y_{1}, m_{2} x_{2}+n_{2} y_{2}, \ldots, m_{l} x_{l}+n_{l} y_{l}, u_{1} x_{1}+v_{1} y_{1}, u_{2} x_{2}+v_{2} y_{2},\right. \\
& \left.\cdots, u_{l} x_{l}+v_{l} y_{l}\right),
\end{aligned}
$$

where $\mu \in[0,1]$ is a parameter, $m_{k}, n_{k}, u_{k}, v_{k}(k=1,2, \ldots, l)$ are chosen constants. We show that the mapping $L(x, y, \mu)$ is a homotopic mapping. Namely, we show when $(x, y)^{T} \in$ $\partial \Omega \cap \operatorname{Ker} L, \mu \in[0,1], L(x, y, \mu) \neq 0$. If when $(x, y)^{T} \in \partial \Omega \cap \operatorname{Ker} L, \mu \in[0,1], L(x, y, \mu)=0$, then for $k=1,2, \ldots, l$,

$$
\begin{aligned}
& (1-\mu)\left(y_{k}-\eta x_{k}\right)+\mu\left(m_{k} x_{k}+n_{k} y_{k}\right)=0, \\
& (1-\mu)\left[-\eta^{2} x_{k}+\eta y_{k}-f_{k}\left(\xi_{k}, y_{k}-\eta x_{k}\right)-g_{k}\left(\xi_{k}, x_{k}\right)+e_{k}\left(\xi_{k}\right)\right. \\
& \left.\quad-\sum_{h=1}^{l} a_{k h}\left(y_{k}-y_{h}\right)\right]+\mu\left(u_{k} x_{k}+v_{k} y_{k}\right)=0 .
\end{aligned}
$$

From (3.5) and (3.6), it follows that

$$
\begin{aligned}
0= & \sum_{k=1}^{l} c_{k}\left\{x_{k}\left[(1-\mu)\left(y_{k}-\eta x_{k}\right)+\mu\left(m_{k} x_{k}+n_{k} y_{k}\right)\right]\right. \\
& +y_{k}\left[( 1 - \mu ) \left(-\eta^{2} x_{k}-f_{k}\left(\xi_{k}, y_{k}-\eta \times x_{k}\right)+g_{k}\left(\xi_{k}, x_{k}\right)+e_{k}\left(\xi_{k}\right)\right.\right. \\
& \left.\left.\left.-\sum_{h=1}^{l} a_{k h}\left(y_{k}-y_{h}\right)\right)+\mu\left(u_{k} x_{k}+v_{k} y_{k}\right)\right]\right\} \\
\leq & \sum_{k=1}^{l} c_{k}\left\{(1-\mu) p_{k}\left(x_{k} y_{k}-\eta x_{k}^{2}\right)+\mu^{*}\left(m_{k} x_{k}^{2}+n_{k} x_{k} y_{k}\right)-(1-\mu) \eta^{2} x_{k} y_{k}\right. \\
& +(1-\mu) \eta y_{k}^{2}+\mu \times\left(v_{k} y_{k}^{2}+u_{k} x_{k} y_{k}\right) \\
& +(1-\mu)\left[-\delta\left(y_{k}-\eta x_{k}\right)^{2}-a\left(y_{k}-\eta x_{k}\right)+\eta r\left|x_{k}\right|\left|y_{k}\right|+\eta^{2} r x_{k}^{2}+\eta e\left|x_{k}\right|\right. \\
& \left.+y_{k}^{2}+0.5\left[b d+b d+\left(\overline{e_{k}}\right)^{2}-\sum_{h=1}^{l} a_{k h}\left(y_{k}^{2}-y_{h}^{2}\right)+\eta e+\eta|a|+|a|\right]\right\} \\
\leq & \sum_{k=1}^{l} c_{k}\left\{\left[-\eta+\left(-\delta \eta^{2}+0.5 b^{2}+0.5 b d+\eta^{2} r-\eta l+0.5 \eta|a|+0.5 \eta e\right)\right.\right. \\
& \left.+\mu\left(m_{k}+\eta-\eta^{2} r+\delta \eta^{2}-0.5 \eta e-0.5 \eta r\right)\right] x_{k}^{2} \\
& +\left[-\eta^{2}+2 \eta \delta+1+\mu\left(l_{k}+u_{k}-1+\eta^{2}-2 \delta \eta\right)\right] x_{k} y_{k} \\
& +\left[(\eta-\delta+0.5 \eta r+0.5|a|+1)+\mu\left(v_{k}-\eta+\delta-1-0.5 \eta l\right)\right] y_{k}^{2}
\end{aligned}
$$




$$
\begin{aligned}
& +0.5\left(\left(\overline{e_{k}}\right)^{2}+b d+d^{2}+\eta e+|a| \eta+|a|\right) \\
& \left.-0.5(1-\mu) \sum_{h=1, k=1}^{l} c_{k} a_{k h}\left(y_{h}^{2}-y_{k}^{2}\right)\right\}
\end{aligned}
$$

from which it follows that, since $\sum_{h=1, k=1}^{l} c_{k} a_{k h}\left(y_{h}^{2}-y_{k}^{2}\right)=0$,

$$
\begin{aligned}
0 \leq & \sum_{k=1}^{l} c_{k}\left\{\left[-\eta-\delta \eta^{2}+0.5 b^{2}+0.5 b d+\eta^{2} r-\eta l+0.5 \eta|a|+0.5 \eta e\right.\right. \\
& \left.+\mu\left(m_{k}+\eta-\eta^{2} r+\delta \eta^{2}-0.5 \eta e-0.5 \eta r\right)\right] x_{k}^{2} \\
& +\left[-\eta^{2}+2 \eta \delta+1+\mu\left(n_{k}+u_{k}-1+\eta^{2}-2 \delta \eta\right)\right] x_{k} y_{k} \\
& +\left[(\eta-\delta+0.5 \eta r+0.5|a|+1)+\mu\left(v_{k}-\eta+\delta-1-0.5 \eta r\right)\right] y_{k}^{2} \\
& \left.+0.5\left(\left(\overline{e_{k}}\right)^{2}+b d+d^{2}+\eta e+|a| \eta+|a|\right)\right\} .
\end{aligned}
$$

Choose $m_{k}, v_{k}, l_{k}, u_{k}$ such that

$$
\begin{aligned}
& m_{k}+\eta-\eta^{2} r+\delta \eta^{2}-0.5 \eta e-0.5 \eta r=0, \\
& n_{k}+u_{k}-1+\eta^{2}-2 \delta \eta=0,
\end{aligned}
$$

and

$$
v_{k}-\eta+\delta-1-0.5 \eta r=0 .
$$

Substituting (3.9)-(3.11) into (3.8) gives

$$
\begin{aligned}
0 \leq & \sum_{k=1}^{l}\left\{\left[-\eta+\left(-\delta \eta^{2}+0.5 b^{2}+0.5 b d+\eta^{2} r+0.5 \eta r+0.5 \eta|a|+0.5 \eta e\right)\right] x_{k}^{2}\right. \\
& +\left(-\eta^{2}+2 \eta \delta+1\right) x_{k} y_{k}+(\eta-\delta+0.5 \eta r+0.5|a|+1) y_{k}^{2} \\
& \left.+0.5\left(\left(\overline{e_{k}}\right)^{2}+b d+d^{2}+\eta e+|a| \eta+|a|\right)\right\} .
\end{aligned}
$$

Since $\left\|(x, y)^{T}\right\|>H$, we have from Remark 1

$$
\begin{aligned}
& \sum_{k=1}^{l}\left\{\left[-\eta+\left(-\delta \eta^{2}+0.5 b^{2}+0.5 b d+\eta^{2} r+0.5 \eta l+0.5 \eta|a|+0.5 \eta e\right)\right]\right. \\
& \quad+x_{k}^{2}\left(-\eta^{2}+2 \eta \delta+1\right) x_{k} y_{k}+(\eta-\delta+0.5 \eta r+0.5|a|+1) y_{k}^{2} \\
& \left.\quad+0.5\left(\left(\overline{e_{k}}\right)^{2}+b d+d^{2}+\eta e+|a| \eta+|a|\right)\right\}<0 .
\end{aligned}
$$

Equation (3.13) contradicts with (3.12). Hence, $L(x, y, \mu)$ is a homotopic mapping, by topological degree theory, we have

$$
\begin{aligned}
& \operatorname{deg}_{B}(J Q N, \partial \Omega \cap \operatorname{Ker} L,(0,0, \ldots, 0)) \\
& \quad=\operatorname{deg}_{B}(L(x, y, 0), \partial \Omega \cap \operatorname{Ker} L,(0,0, \ldots, 0))
\end{aligned}
$$




$$
\begin{aligned}
& =\operatorname{deg}_{B}(L(x, y, 1), \partial \Omega \cap \operatorname{Ker} L,(0,0, \ldots, 0)) \\
& =\operatorname{deg}_{B}\left(m_{1} x_{1}+n_{1} y_{1}, m_{2} x_{2}+n_{2} y_{2}, \ldots, m_{l} x_{l}+n_{l} y_{l}, u_{1} x_{1}+v_{1} y_{1}, \ldots, u_{l} x_{l}+v_{l} y_{l}\right) \\
& =\operatorname{sign}\left|\begin{array}{cc}
E & F \\
M & N
\end{array}\right|,
\end{aligned}
$$

where

$$
\begin{aligned}
& E=\operatorname{diag}\left(m_{1}, m_{2}, \ldots, m_{l}\right), \quad F=\operatorname{diag}\left(n_{1}, n_{2}, \ldots, n_{l}\right), \\
& M=\operatorname{diag}\left(u_{1}, u_{2}, \ldots, u_{l}\right), \quad N=\operatorname{diag}\left(v_{1}, v_{2}, \ldots, v_{l}\right) .
\end{aligned}
$$

Since

$$
\left|\begin{array}{ll}
E & F \\
M & N
\end{array}\right|=|E M-F N|=\prod_{k=1}^{l}\left(m_{k} v_{k}-l_{k} u_{k}\right) .
$$

Then substituting (3.15) into (3.14) gives

$$
\begin{aligned}
& \operatorname{deg}_{B}(J Q N, \partial \Omega \cap \operatorname{Ker} L,(0,0, \ldots, 0)) \\
& \quad=\operatorname{sign} \prod_{k=1}^{l}\left(m_{k} v_{k}-n_{k} u_{k}\right) \\
& \quad=\operatorname{sign} \prod_{k=1}^{l}\left(\left(-\eta+\eta^{2} r-\delta \eta^{2}+0.5 \eta e+0.5 \eta l\right)(\eta-\delta+1+0.5 \eta r)-n_{k} u_{k}\right) .
\end{aligned}
$$

Again choose $n_{k}, u_{k}$ such that

$$
n_{k} u_{k} \neq\left(-\eta+\eta^{2} r-\delta \eta^{2}+0.5 \eta e+0.5 \eta r\right)(\eta-\delta+1+0.5 \eta r) \text {. }
$$

Then

$$
\operatorname{deg}_{B}(J Q N, \partial \Omega \cap \operatorname{Ker} L,(0,0, \ldots, 0)) \neq 0 .
$$

By Lemma 2.1, system (1.7) has at least an $\omega$-periodic solution. This completes the proof of Theorem 3.1.

Remark 2 In the proof of Theorem 3.1, $m_{k}, n_{k}, u_{k}, v_{k}$ are chosen such that $m_{k}+\eta-\eta^{2} r+$ $\delta \eta^{2}-0.5 \eta e-0.5 \eta r=0, n_{k}+u_{k}-1+\eta^{2}-2 \delta \eta=0, v_{k}-\eta+\delta-1-0.5 \eta r=0, n_{k} u_{k} \neq(-\eta+$ $\left.\eta^{2} r-\delta \eta^{2}+0.5 \eta e+0.5 \eta r\right)(\eta-\delta+1+0.5 \eta r)$. Such $n_{k}, u_{k}$ indeed exist, for example, letting $\delta=-1, \eta=0.5, b=d=0.001,|a|=0.003, r=3, e=0.003$, then $v_{k}=3.25, m_{k}=1.25075, n_{k}$, $u_{k}$ satisfy $n_{k}+u_{k}=-0.25, n_{k} u_{k} \neq 3.25 \times 1.25075$. Thus by taking $n_{k}=-0.5, u_{k}=0.25$, the task can be fulfilled.

Remark 3 In our Theorem 3.1, conditions $\left(A_{4}\right)$ and $\left(A_{5}\right)$ in Theorem 1 in [21] are removed and conditions $\left(A_{1}\right)$ and $\left(A_{2}\right)$ in Theorem 1 in [21] are replaced with conditions $\left(H_{1}\right)$ and $\left(H_{2}\right)$. Hence, our result on the existence of periodic solutions for a coupled Rayleigh system is different from that obtained in Theorem 1 in [21]. 
Theorem 3.2 Under $\left(A_{1}\right)-\left(A_{3}\right)$, system (1.7) has at least one $\omega$-periodic solution.

Proof Define the same $X, Z, G_{k}, F_{k}, L, N, P$, and $Q$ as those in the proof of Theorem 3.1, where the norm of $X$ is different from that of $X$ in the proof of Theorem 3.1. Here, we define the norm of $X$ by $\|x\|=\left(\sum_{k=1}^{l} \max _{t \in[0, \omega]}\left[\left|x_{k}(t)\right|^{2}+\left|y_{k}(t)\right|^{2}\right]\right)^{\frac{1}{2}}$.

Clearly, $Q N$ and $K_{P}(I-Q) N$ are continuous and $Q N(\bar{\Omega})$ is bounded, where $\Omega$ is an open set in $X$. Then by Arzela-Ascoli theorem, we can prove that $\overline{K_{P}(I-Q) N(\bar{\Omega})}$ is compact. Hence, $N$ is $L$-compact on $\bar{\Omega}$.

Corresponding to the operator equation $L x=\lambda N x, \lambda \in(0,1)$, we have system (2.1), a.e., system (4) in Lemma 3 of [21]. By Lemma 3 in [21], for every periodic solution $(x(t), y(t))^{T}=$ $\left(x_{1}(t), x_{2}(t), \ldots, x_{l}(t), y_{1}(t), \ldots, y_{l}(t)\right)^{T}$ of $L z=\lambda N z$, there is $H>0$, which is independent of the choice of $\lambda$ such that $\left\|(x(t), y(t))^{T}\right\|<H$. We set $\Omega=\left\{(x(t), y(t))^{T} \in X:\left\|(x, y)^{T}\right\|<H+\right.$ $r$, where $r>0$ is a chosen positive constant such that the bound of $\Omega$ is larger. Hence, for any $\lambda \in(0,1), z \in \partial \Omega \cap \operatorname{Dom} L, L z \neq \lambda N z$. When $z \in \partial \Omega \cap \operatorname{Ker} P$, we will show $Q N z \neq 0$. When $z \in \partial \Omega \cap \operatorname{Ker} L, z \in R^{2 l}$ (namely $z$ is a constant vector) with $\|z\|=\left\|(x, y)^{T}\right\|=H+r$. If $z$ is a constant vector with $\|z\|=H+r, Q N z=0$, then it follows that the constant vector $z$ with $\|z\|=H+r$ satisfies, for $k=1,2, \ldots, l$,

$$
\frac{1}{\omega} \int_{0}^{\omega} G_{k}(t) d t=0, \quad \frac{1}{\omega} \int_{0}^{\omega} F_{k}(t) d t=0
$$

Hence, there exist $t_{k}(i=1,2), \xi_{i} \in[0, \omega](k=1,2, \ldots, l)$ such that

$$
G_{k}\left(t_{k}\right)=0, \quad F_{k}\left(\xi_{k}\right)=0 .
$$

From (3.16), we have

$$
0=\sum_{k=1}^{l} c_{k}\left(x_{k} G_{k}\left(t_{k}\right)+y_{k} F_{k}\left(\xi_{k}\right)\right) .
$$

From the proof of page 4 in Lemma 3 of [21], it follows from (3.17) that

$$
\begin{aligned}
0= & \sum_{k=1}^{l}\left(x_{k} G_{k}\left(t_{k}\right)+y_{k} F_{k}\left(\xi_{k}\right)\right) \\
= & \sum_{k=1}^{l}\left\{-\eta x_{k}^{2}(t)-\eta^{2} x_{k}(t) y_{k}(t)+\eta y_{k}^{2}(t)-y_{k}(t) f_{k}\left(t, y_{k}(t)-\eta x_{k}(t)\right)\right. \\
& \left.+y_{k}(t)\left[x_{k}(t)-g_{k}\left(t, x_{k}(t)\right)\right]+y_{k}(t) e_{k}(t)-\sum_{h=1}^{l} a_{k h} y_{k}(t)\left[y_{k}(t)-y_{h}(t)\right]\right\} \\
< & \sum_{k=1}^{l} c_{k}\left[-\frac{\eta^{2}}{4} x_{k}^{2}-\frac{\eta^{2}}{2} y_{k}^{2}+\left(\overline{e_{k}}\right)^{2}\right]<0 .
\end{aligned}
$$

This a contradiction. Hence, for each $z \in \partial \cap \operatorname{Ker} L, Q N z \neq 0$. 
Finally, we show that $\operatorname{deg}_{B}\{J Q N, \Omega \cap \operatorname{Ker} L,(0,0, \ldots, 0)\} \neq 0$. We only show that $\operatorname{deg}_{B}\{J Q N z, \Omega \cap \operatorname{Ker} L,(0,0, \ldots, 0)\} \neq 0$ when $z \in \partial \Omega \cap \operatorname{Ker} L$. To this end, we construct the following mapping for $k=1,2, \ldots, l$ :

$$
\begin{aligned}
L_{1}\left(x, y, \mu^{*}\right) & \\
= & \left(1-\mu^{*}\right)\left[y_{1}-\eta x_{1}, y_{2}-\eta x_{2}, \ldots, y_{n}-\eta x_{n},\right. \\
& -\eta^{2} x_{1}+\eta y_{1}-f_{1}\left(\xi_{1}, y_{1}-\eta x_{1}\right)-g_{1}\left(\xi_{1}, x_{1}\right)+e_{1}\left(\xi_{1}\right)-\sum_{h=1}^{l} a_{1 h}\left(y_{1}-y_{h}\right), \\
& -\eta^{2} x_{2}+\eta y_{2}-f_{2}\left(\xi_{2}, y_{2}-\eta x_{2}\right)-g_{2}\left(\xi_{2}, x_{2}\right)-\sum_{h=1}^{l} a_{2 h}\left(y_{2}-y_{h}\right)+e_{2}\left(\xi_{2}\right), \ldots, \\
& \left.-\eta^{2} x_{l}+\eta y_{l}-f_{l}\left(\xi_{l}, y_{l}-\eta x_{l}\right)-g_{l}\left(\xi_{l}, x_{l}\right)+e_{l}\left(\xi_{l}\right)-\sum_{h=1}^{l} a_{l h}\left(y_{l}-y_{h}\right)\right] \\
& +\mu^{*} \times\left(m_{1}^{*} x_{1}+n_{1}^{*} y_{1}, m_{2}^{*} x_{2}+n_{2}^{*} y_{2}, \ldots, m_{l}^{*} x_{l}+n_{l}^{*} y_{l}, u_{1}^{*} x_{1}+v_{1}^{*} y_{1},\right. \\
& \left.u_{2}^{*} x_{2}+v_{2}^{*} y_{2}, \ldots, u_{l}^{*} x_{l}+v_{l}^{*} y_{l}\right),
\end{aligned}
$$

where $\mu^{*} \in[0,1]$ is a parameter, $m_{k}^{*}, r_{k}^{*}, u_{k}^{*}, v_{k}^{*}(k=1,2, \ldots, l)$ are chosen constants. We show that the mapping $L_{1}\left(x, y, \mu^{*}\right)$ is a homotopic mapping. Namely, we show when $(x, y)^{T} \in \partial \Omega \cap \operatorname{Ker} L, \mu^{*} \in[0,1], L_{1}\left(x, y, \mu^{*}\right) \neq 0$. If when $(x, y)^{T} \in \partial \Omega \cap \operatorname{Ker} L, \mu^{*} \in[0,1]$, $L_{1}\left(x, y, \mu^{*}\right)=0$, then for $k=1,2, \ldots, l$,

$$
\begin{aligned}
& \left(1-\mu^{*}\right)\left(y_{k}-\eta x_{k}\right)+\mu^{*}\left(m_{k}^{*} x_{k}+n_{k}^{*} y_{k}\right)=0, \\
& \left(1-\mu^{*}\right)\left[-\eta^{2} x_{k}+\eta y_{k}-f_{k}\left(\xi_{k}, y_{k}-\eta x_{k}\right)-g_{k}\left(\xi_{k}, x_{k}\right)+e_{k}\left(\xi_{k}\right)\right. \\
& \left.\quad-\sum_{h=1}^{l} a_{k h}\left(y_{k}-y_{h}\right)\right]+\mu^{*}\left(u_{k}^{*} x_{k}+v_{k}^{*} y_{k}\right)=0 .
\end{aligned}
$$

From (3.19) and (3.20), it follows that

$$
\begin{aligned}
0= & \sum_{k=1}^{l} c_{k}\left\{x_{k}\left[\left(1-\mu^{*}\right)\left(y_{k}-\eta x_{k}\right)+\mu^{*}\left(m_{k}^{*} x_{k}+l_{k}^{*} y_{k}\right)\right]\right. \\
& +y_{k}\left[( 1 - \mu ^ { * } ) \left(-\eta^{2} x_{k}+\eta y_{k}-f_{k}\left(\xi_{k}, y_{k}-\eta x_{k}\right)-g_{k}\left(\xi_{k}, x_{k}\right)+e_{k}\left(\xi_{k}\right)\right.\right. \\
& \left.\left.\left.-\sum_{h=1}^{l} a_{k h}\left(y_{k}-y_{h}\right)\right)+\mu^{*}\left(u_{k}^{*} x_{k}+v_{k}^{*} y_{k}\right)\right]\right\} .
\end{aligned}
$$


Let $f_{k}\left(\xi_{k}, y_{k}-\eta x_{k}\right)=\left(y_{k}-\eta x_{k}\right) \beta_{k}\left(\xi_{k}, y_{k}-\eta x_{k}\right)$, then from $\left(A_{2}\right)$ we can easily obtain that $0<\frac{2(\eta+1)}{2-\eta} \leq \beta_{k}\left(\xi_{k}, y_{k}-\eta x_{k}\right) \leq 2$. By using $\left(A_{1}\right),\left(A_{2}\right)$, we have from (3.21)

$$
\begin{aligned}
0= & \sum_{k=1}^{l} c_{k}\left\{\left(1-\mu^{*}\right)\left(x_{k} y_{k}-\eta x_{k}^{2}\right)+\mu^{*}\left(m_{k}^{*} x_{k}^{2}+n_{k}^{*} x_{k} y_{k}\right)\right. \\
& +\left(1-\mu^{*}\right)\left(-\eta^{2} x_{k} y_{k}+\eta y_{k}^{2}-y_{k}^{2} \beta_{k}\left(\xi_{k}, y_{k}-\eta x_{k}\right)\right. \\
& +\eta x_{k} y_{k} \beta_{k}\left(\xi_{k}, y_{k}-\eta x_{k}\right)+y_{k}\left[x_{k}(t)-g_{k}\left(\xi_{k}, x_{k}\right)\right]-x_{k} y_{k}+e_{k}\left(\xi_{k}\right) \times y_{k} \\
& \left.\left.-\sum_{h=1}^{l} a_{k h}\left(y_{k}^{2}-y_{h} y_{k}\right)\right)+\mu^{*}\left(u_{k}^{*} x_{k} y_{k}+v_{k}^{*} y_{k}^{2}\right)\right\} \\
\leq & \sum_{k=1}^{l} c_{k}\left\{\left(1-\mu^{*}\right)\left(x_{k} y_{k}-\eta x_{k}^{2}\right)\right. \\
& +\mu^{*}\left(m_{k}^{*} x_{k}^{2}+n_{k}^{*} x_{k} y_{k}\right)+\left(1-\mu^{*}\right)\left(-\eta^{2} x_{k} y_{k}+\eta y_{k}^{2}-y_{k}^{2} \times \beta_{k}\left(\xi_{k}, y_{k}-\eta x_{k}\right)\right. \\
& +\eta x_{k} y_{k} \beta_{k}\left(\xi_{k}, y_{k}-\eta x_{k}\right)+y_{k}^{2}+0.5\left[x_{k}(t)-g_{k}\left(\xi_{k}, x_{k}\right)\right]^{2}-x_{k} y_{k} \\
& \left.\left.+0.5 \times e_{k}^{2}\left(\xi_{k}\right)+0.5 \sum_{h=1}^{l} a_{k h}\left(y_{h}^{2}-y_{k}^{2}\right)\right)+\mu^{*}\left(u_{k}^{*} x_{k} y_{k}+v_{k}^{*} y_{k}^{2}\right)\right\}
\end{aligned}
$$

from which it follows that noting $\sum_{k=1}^{l} c_{k} a_{k h}\left(y_{h}^{2}-y_{k}^{2}\right)=0$,

$$
\begin{aligned}
0 \leq & \sum_{k=1}^{l} c_{k}\left\{\left(1-\mu^{*}\right)\left(x_{k} y_{k}-\eta x_{k}^{2}\right)+\mu^{*}\left(m_{k}^{*} x_{k}^{2}+\eta_{k}^{*} x_{k} y_{k}\right)\right. \\
& +\left(1-\mu^{*}\right)\left(-\eta^{2} x_{k} y_{k}+\eta y_{k}^{2}-y_{k}^{2} \times \beta_{k}\left(\xi_{k}, y_{k}-\eta x_{k}\right)\right. \\
& +\eta x_{k} y_{k} \beta_{k}\left(\xi_{k}, y_{k}-\eta x_{k}\right)+y_{k}^{2}+0.5 x_{k}^{2}-\delta x_{k}^{2}+0.5 \mu_{1} x_{k}^{2}-x_{k} y_{k}+0.5 \\
& \left.\left.\times e_{k}^{2}\left(\xi_{k}\right)\right)+\mu^{*}\left(u_{k}^{*} x_{k} y_{k}+v_{k}^{*} y_{k}^{2}\right)\right\} \\
= & \sum_{k=1}^{l} c_{k}\left\{\left(\eta\left(\beta_{k}\left(\xi_{k}, y_{k}-\eta x_{k}\right)-\eta\right)+\mu^{*}\left[n_{k}^{*}+\eta\left(\eta-\beta_{k}\left(\xi_{k}, y_{k}-\eta x_{k}\right)\right)+u_{k}^{*}\right]\right) x_{k} y_{k}\right. \\
& +\left[0.5-\delta+0.5 \mu_{1}-\eta+\mu^{*}\left(m_{k}^{*}+\eta-0.5+\delta-0.5 \mu_{1}\right)\right] x_{k}^{2} \\
& +\left(\eta-\beta_{k}\left(\xi_{k}, y_{k}-\eta x_{k}\right)+1+\mu^{*}\left(-\eta \beta_{k}\left(\xi_{k}, y_{k}-\eta x_{k}\right)-1+v_{k}^{*}\right)\right) y_{k}^{2} \\
& \left.+0.5\left(\overline{e_{k}}\right)^{2}\right\} .
\end{aligned}
$$

Noting that

$$
\begin{aligned}
& \eta\left[\beta_{k}\left(\xi_{k}, y_{k}-\eta x_{k}\right)-\eta\right] \geq \eta\left(\frac{2(\eta+1)}{2-\eta}-\eta\right)=\eta\left(\frac{\eta^{2}+2}{2-\eta}\right)>0, \\
& \eta\left[\beta_{k}\left(\xi_{k}, y_{k}-\eta x_{k}\right)-\eta\right] x_{k} y_{k} \leq 0.5 \eta\left[\beta_{k}\left(\xi_{k}, y_{k}-\eta x_{k}\right)-\eta\right]\left(x_{k}^{2}+y_{k}^{2}\right)
\end{aligned}
$$


and

$$
\begin{aligned}
\mu^{*} & \left\{n_{k}^{*}+u_{k}^{*}+\eta\left[\eta-\beta_{k}\left(\xi_{k}, y_{k}-\eta x_{k}\right)\right]\right\} x_{k} y_{k} \\
& \leq 0.5 \mu^{*}\left\{\left|n_{k}^{*}\right|+\left|u_{k}^{*}\right|+\eta\left[\beta_{k}\left(\xi_{k}, y_{k}-\eta x_{k}\right)-\eta\right]\right\}\left(x_{k}^{2}+y_{k}^{2}\right)
\end{aligned}
$$

it follows from (3.22) that

$$
\begin{aligned}
0 \leq & \sum_{k=1}^{l} c_{k}\left\{\left(0.5-\delta+0.5 \mu_{1}-\eta+0.5 \eta\left[\beta_{k}\left(\xi_{k}, y_{k}-\eta x_{k}\right)-\eta\right]\right.\right. \\
& \left.+\mu^{*}\left[m_{k}^{*}+\eta-0.5+\delta-0.5 \mu_{1}+0.5\left(\left|n_{k}^{*}\right|+\left|u_{k}^{*}\right|\right)+0.5 \eta\left[\beta_{k}\left(\xi_{k}, y_{k}-\eta x_{k}\right)-\eta\right]\right]\right) x_{k}^{2} \\
& +\left(0.5 \eta\left[\beta_{k}\left(\xi_{k}, y_{k}-\eta x_{k}\right)-\eta\right]+\eta+1-\beta_{k}\left(\xi_{k}, y_{k}-\eta x_{k}\right)\right. \\
& \left.+\mu^{*}\left[-\eta \beta_{k}\left(\xi_{k}, y_{k}-\eta x_{k}\right)-1+v_{k}^{*}+0.5\left(\left|n_{k}^{*}\right|+\left|u_{k}^{*}\right|\right)+\eta\left[-\eta \beta_{k}\left(\xi_{k}, y_{k}-\eta x_{k}\right)\right]\right]\right) y_{k}^{2} \\
& \left.+0.5\left(\overline{e_{k}}\right)^{2}\right\} \\
= & \sum_{k=1}^{l} c_{k}\left\{\left[-\frac{1}{2}\left(2 \delta-\mu_{1}-1+\eta^{2}+\eta\left[2-\beta_{k}\left(\xi_{k}, y_{k}-\eta x_{k}\right)\right]\right)\right.\right. \\
& \left.-\frac{1}{2} \mu^{*}\left(-2 m_{k}^{*}-4 \eta+1-2 \delta+\mu_{1}+\eta^{2}-\left|n_{k}^{*}\right|-\left|u_{k}^{*}\right|+\eta\left[2-\beta_{k}\left(\xi_{k}, y_{k}-\eta x_{k}\right)\right]\right)\right] x_{k}^{2} \\
& +\left[-\frac{1}{2}\left(\eta^{2}+(2-\eta) \beta_{k}\left(\xi_{k}, y_{k}-\eta x_{k}\right)-2-2 \eta\right)+\mu^{*}\left(-\eta \beta_{k}\left(\xi_{k}, y_{k}-\eta x_{k}\right)-1\right.\right. \\
& \left.\left.\left.+v_{k}^{*}+0.5\left(\left|n_{k}^{*}+\right| u_{k}^{*} \mid\right)+\eta\left[\beta_{k}\left(\xi_{k}, y_{k}-\eta x_{k}\right)-\eta\right]\right)\right] y_{k}^{2}+\left(\overline{e_{k}}\right)^{2}\right\} \\
\leq & \sum_{k=1}^{l} c_{k}\left\{-\frac{\eta^{2}}{4} x_{k}^{2}-\frac{\eta^{2}}{2} y_{k}^{2}+\left(\overline{e_{k}}\right)^{2}-\frac{1}{2} \mu^{*}\left(-2 m_{k}^{*}-4 \eta+1-2 \delta+\mu_{1}+\eta^{2}\right.\right. \\
& \left.\left.-\left|n_{k}^{*}\right|-\mid u_{k}^{*}\right) \mid x_{k}^{2}-\frac{1}{2} \mu^{*}\left(2-2 v_{k}^{*}-\left|n_{k}^{*}\right|-\left|u_{k}^{*}\right|+2 \eta^{2}\right) y_{k}^{2}\right\} .
\end{aligned}
$$

Choose $v_{k}^{*}, m_{k}^{*}, u_{k}^{*}, n_{k}^{*}$ such that

$$
2 v_{k}^{*}=2-\left|n_{k}^{*}\right|-\left|u_{k}^{*}\right|+2 \eta^{2}
$$

and

$$
2 m_{k}^{*}=1-4 \eta-2 \delta+\mu_{1}+\eta^{2}-\left|n_{k}^{*}\right|-\left|u_{k}^{*}\right| .
$$

Substituting (3.25) and (3.26) into (3.24) gives

$$
0 \leq \sum_{k=1}^{l} c_{k}\left[-\frac{\eta^{2}}{4} x_{k}^{2}-\frac{\eta^{2}}{2} y_{k}^{2}+\left(\overline{e_{k}}\right)^{2}\right]
$$

From the proof of Lemma 3 in [21], we have

$$
\sum_{k=1}^{l} c_{k}\left[-\frac{\eta^{2}}{4} x_{k}^{2}-\frac{\eta^{2}}{2} y_{k}^{2}+\left(\overline{e_{k}}\right)^{2}\right]<0 .
$$


The rest of the proof is similar to that of the corresponding part in Theorem 3.1, and it is omitted.

Remark 4 In our Theorem 3.2, conditions $\left(A_{4}\right)$ and $\left(A_{5}\right)$ in Theorem 1 in [21] are removed, the remaining conditions $\left(A_{1}\right)-\left(A_{3}\right)$ are the same. Hence, our result improves Theorem 1 in [21].

Remark 5 By applying new inequality techniques, we establish new sufficient conditions for the existence of periodic solutions of a coupled Rayleigh system. Our method can be applied to studying the existence of periodic solutions for any second-order differential system.

\section{Numerical test}

Example 1 Consider the following Rayleigh system:

$$
\left\{\begin{aligned}
x_{k}^{\prime}(t)= & y_{k}(t)-\eta x_{k}(t), \\
y_{k}^{\prime}(t)= & -\eta^{2} x_{k}(t)+\eta y_{k}(t)-f_{k}\left(t, y_{k}(t)-\eta x_{k}(t)\right)-g_{k}\left(t, x_{k}(t)\right) \\
& +e_{k}(t)-\sum_{h=1}^{l} a_{k h}\left[y_{k}(t)-y_{h}(t)\right],
\end{aligned}\right.
$$

where $k=1,2,3,4, \eta=0.4$ and $g_{k}\left(t, x_{k}(t)\right)=0.05\left|x_{k}(t)\right|+0.05 \cos x_{k}(t)+0.05 \sin x_{k}$, $f_{k}\left(t, x_{k}(t)\right)=\left(0.5+0.6 \sin x_{k}(t)\right) x_{k}(t)+0.003, e_{k}(t)=1+\cos t$.

We can check that $\left|g_{k}\left(t, x_{k}\right)\right| \leq 0.05\left|x_{k}(t)\right|+0.06$, and we take $b=0.005, d=0.06$. $x_{k} f_{k}\left(t, x_{k}\right) \geq-0.1 x_{k}^{2}+0.03 x_{k}$, and $\delta=-0.1, a=0.03$. Taking $\eta=0.4$, we get $A=-\eta-\delta \eta^{2}+$ $0.5 b^{2}+0.5 b d+\eta^{2}+0.5 \eta r+0.5 \eta|a|+0.5 \eta e<0$, thus conditions $\left(H_{1}\right),\left(H_{2}\right)$ are satisfied.

Since $g_{k}(t, x)$ is not differential in $x_{k}$, thus condition $\left(A_{4}\right)$ in [21] cannot be satisfied; since $\int_{0}^{1}(1+\cos t) d t \neq 0$, hence condition $\left(A_{5}\right)$ in [21] cannot be satisfied, hence the existence of periodic solutions of system (4.1) cannot be verified by these results in [21]. Assuming that

$$
B=\left(a_{k h}\right)_{4 \times 4}=\left(\begin{array}{cccc}
0 & 2 & 0.6 & 1 \\
0.3 & 0 & 3 & 0.4 \\
3 & 0.5 & 0 & 2 \\
2 & 0.6 & 2 & 0
\end{array}\right) \text {, }
$$

Figure 1 The phrase plan $\left(x_{1}(t), y_{1}(t)\right)$

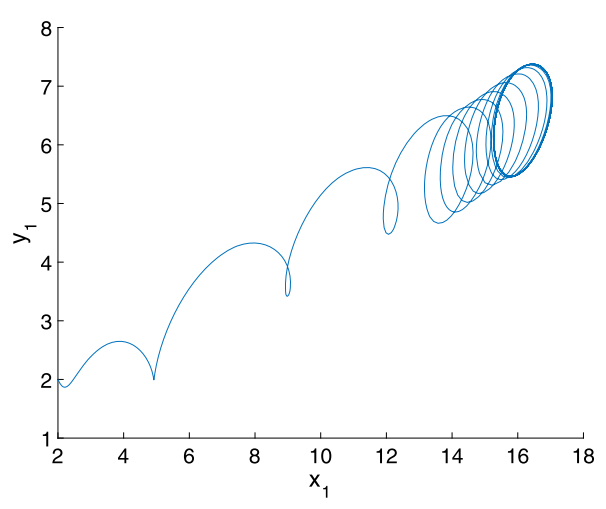


Figure 2 The phrase plan $\left(x_{2}(t), y_{2}(t)\right)$

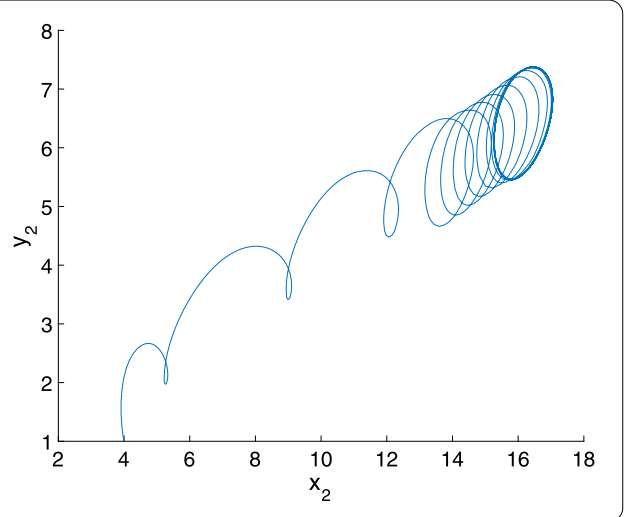

Figure 3 The phrase plan $\left(x_{3}(t), y_{3}(t)\right)$

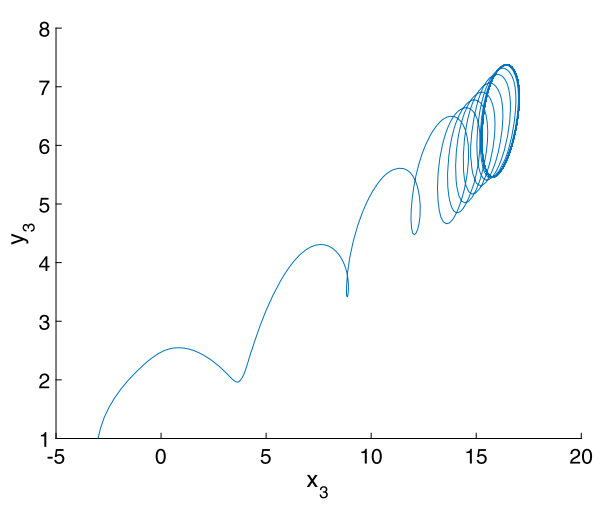

Figure 4 The phrase plan $\left(x_{4}(t), y_{4}(t)\right)$

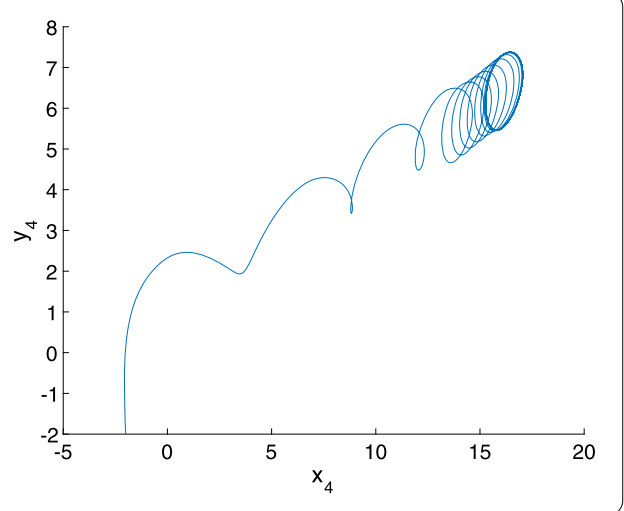

we can check that condition $\left(A_{3}\right)$ holds. Now, all the conditions in Theorem 3.1 in our paper are satisfied. The solution of system (4.1) is shown in Figs. 1-4, from which we can clearly see that system (4.1) has at least one periodic solution.

Example 2 In system (4.1), we set $g_{k}\left(t, x_{k}(t)\right)=\left(1+0.001\left|\sin x_{k}(t)\right|+0.001 \sin t\right) x_{k}(t)$, $f_{k}\left(t, x_{k}(t)\right)=0.2 x_{k}(t) \sin 2 t, e_{k}(t)=\sin t+1$. It is easy to verify that $\left(A_{1}\right),\left(A_{2}\right)$, and $\left(A_{3}\right)$ are 
Figure 5 The phrase plan $\left(x_{1}(t), y_{1}(t)\right)$

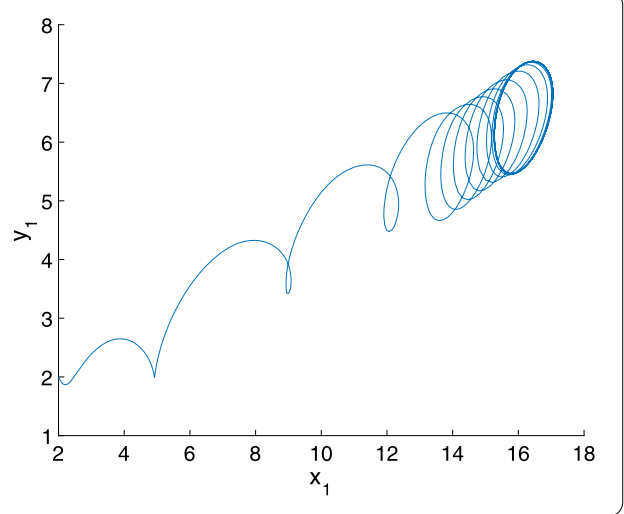

Figure 6 The phrase plan $\left(x_{2}(t), y_{2}(t)\right)$

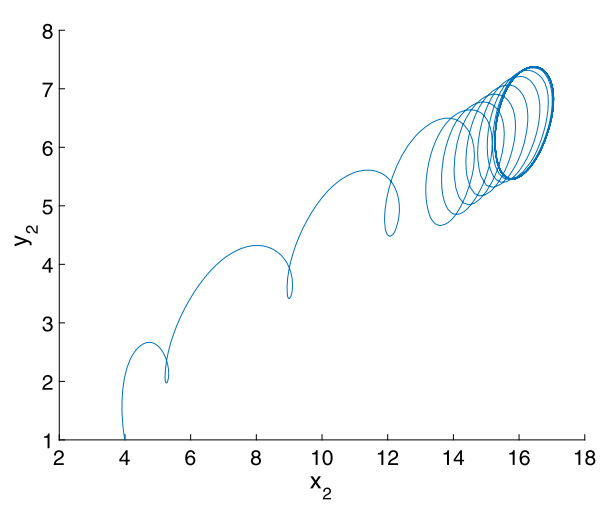

satisfied assuming that

$$
B=\left(a_{k h}\right)_{4 \times 4}=\left(\begin{array}{cccc}
0 & 2 & 6 & 1 \\
0.3 & 0 & 1 & 0.4 \\
3 & 0.5 & 0 & 2 \\
2 & 0.6 & 2 & 0
\end{array}\right) .
$$

But $\left(A_{4}\right)$ is not satisfied since $m_{k}\left(x_{k}\right)$ contains $\left|\sin x_{k}(t)\right|$, which is not differential. Hence, the existence of periodic solutions of system (4.1) cannot be verified by the results in [21]. On the other hand, by our Theorem 3.1, system (4.1) has at least one $\omega$-periodic solution.

The solution of system (4.1) is shown in Figs. 5-8, from which we can clearly see that system (4.1) has at least one periodic solution.

\section{Conclusion}

In the paper, we discuss the existence of periodic solutions for a class of coupled Rayleigh systems by combining graph theory with continuation theorem as well as Lyapunov functions. By the above study methods and by using novel inequality techniques, we obtain new sufficient conditions to ensure the existence of periodic solutions for system (1.7). Our results and method are completely new. 
Figure 7 The phrase plan $\left(x_{3}(t), y_{3}(t)\right)$

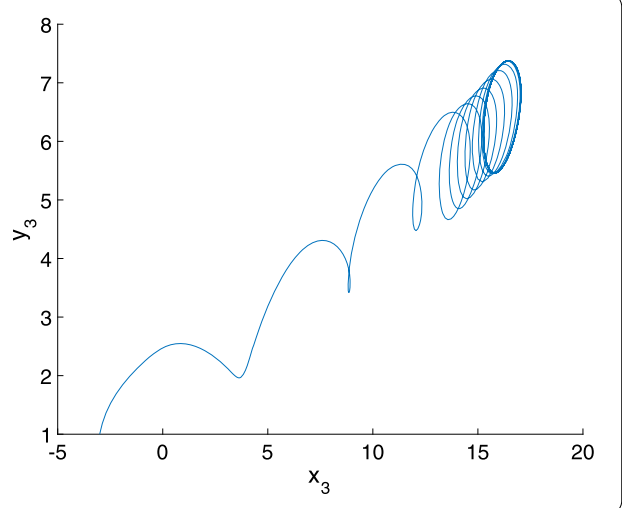

Figure 8 The phrase plan $\left(x_{4}(t), y_{4}(t)\right)$

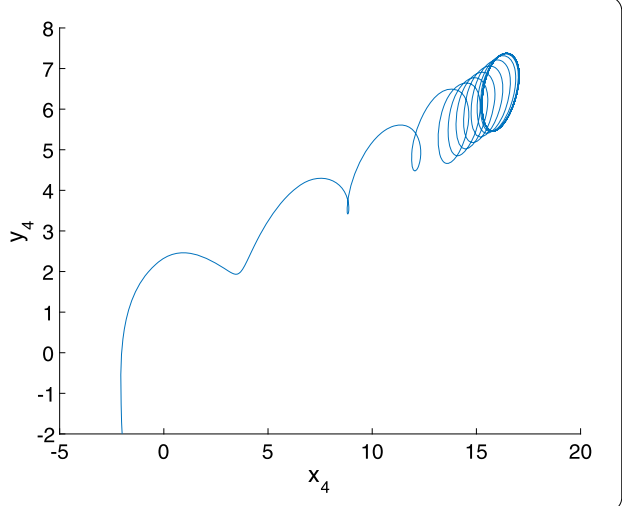

\section{Acknowledgements}

The authors would like to thank the handling editors and the anonymous reviewers.

Funding

None.

\section{Competing interests}

The authors declare that they have no competing interests.

\section{Authors' contributions}

All authors read and approved the final manuscript.

\section{Author details}

'School of Applied Mathematical Science, Xiamen University of Technology, Xiamen, China. ${ }^{2}$ Department of

Mathematics and Computer Science, Nanchang Normal University, Nanchang, China. ${ }^{3}$ College of Mathematics and Econometrics, Hunan University, Changsha, China.

\section{Publisher's Note}

Springer Nature remains neutral with regard to jurisdictional claims in published maps and institutional affiliations.

Received: 5 May 2019 Accepted: 15 July 2019 Published online: 09 August 2019

\section{References}

1. Li, X., Ma, Q.: Boundedness of solutions for second order differential equations with asymmetric nonlinearity. J. Math. Anal. 314, 233-253 (2006)

2. Radhakrishnan, S.: Exact solutions of Rayleigh's equation and sufficient conditions for inviscid instability of parallel, boundedness shear flows. Z. Angew. Math. Phys. 45, 615-637 (1994)

3. Habets, P., Torres, P.J.: Some multiplicity results for periodic solutions of a Rayleigh differential equation. Dyn. Contin. Discrete Impuls. Syst., Ser. A Math. Anal. 8, 335-351 (2001)

4. Cao, H., Liu, B.: Existence and uniqueness of periodic solutions for Rayleigh-type equations. Appl. Math. Comput. 211, 148-154 (2009)

5. Lu, S., Ge, W.: Some new results on the existence of periodic solutions to a kind of Rayleigh equation with a deviating argument. Nonlinear Anal., Theory Methods Appl. 56, 501-514 (2004) 
6. Ma, T.: Periodic solutions of Rayleigh equations via time-maps. Nonlinear Anal., Theory Methods Appl. 75, 4137-4144 (2012)

7. Wang, Y., Zhang, L.: Existence of asymptotically stable periodic solutions of a Rayleigh type equation. Nonlinear Anal., Theory Methods Appl. 71, 1728-1735 (2009)

8. Lord, J.W., Strutt, R.: Theory of Sound, vol. 1. Dover, New York (1877, re-issued 1945)

9. Zhang, Z.Q., Wang, L.P.: Existence and global exponential stability of a periodic solution to discrete-time Cohen-Grossberg BAM neural networks with delays. J. Korean Math. Soc. 48(4), 727-747 (2011)

10. Zhang, Z.Q., Cao, J.D.: Periodic solutions for complex-valued neural networks of neutral type by combining graph theory with coincidence degree theory. Adv. Differ. Equ. 2018, 261 (2018)

11. Zhang, Z.Q., Liu, K.Y.: Existence and global exponential stability of a periodic solution to interval general bidirectional associative memory (BAM) neural networks with multiple delays on time scales. Neural Netw. 24, 427-439 (2011)

12. Zhang, Z.Q., Zheng, T.: Global asymptotic stability of periodic solutions for delayed complex-valued Cohen-Grossberg neural networks by combining coincidence degree theory with LMI method. Neurocomputing 289, 220-230 (2018)

13. Liu, K.Y., Zhang, Z.Q., Wang, L.P.: Existence and global exponential stability of periodic solution to Cohen-Grossberg BAM neural networks with time-varying delays. Abstr. Appl. Anal. 2012, Article ID 805846 (2012)

14. Hu, D.W., Zhang, Z.Q.: Four positive periodic solutions to a Lotka-Volterra cooperative system with harvesting terms. Nonlinear Anal., Real World Appl. 11,1115-1121 (2010)

15. Zhang, Z.Q., Hou, Z.T.: Existence of four positive periodic solutions for a ratio-dependent predator-prey system with multiple exploited (or harvesting) terms. Nonlinear Anal., Real World Appl. 11, 1560-1571 (2010)

16. Gao, S., Li, S.S., Wu, B.Y.: Periodic solutions of discrete time periodic time-varying coupled system on networks. Chaos Solitons Fractals 103, 246-255 (2017)

17. Suo, J.H., Sun, J.T., Zhang, Y.: Stability analysis for impulsive coupled system on networks. Neurocomputing 99 $172-177(2013)$

18. Zhang, X.H., Li, W.X., Wang, K.: The existence of periodic solutions for coupled system on networks with time delays. Neurocomputing 152, 287-293 (2015)

19. Zhang, X.H., Li, W.X., Wang, K.: Periodic solutions of coupled systems on networks with both time-delay and linear coupling. IMA J. Appl. Math. 80, 1871-1889 (2015)

20. Zhang, X.H., Li, W.X., Wang, K.: The existence and global exponential stability of periodic solution for a neutral coupled system on networks with delays. Appl. Math. Comput. 264, 208-217 (2015)

21. Guo, Y., Liu, S., Ding, X.H.: The existence of periodic solutions for coupled Rayleigh. Neurocomputing 191, 398-408 (2016)

22. Yang, X., Lu, J.: Finite-time synchronization of coupled networks with Markovian topology and impulsive effects. IEEE Trans. Autom. Control 61, 2256-2261 (2016)

23. Li, M.Y., Shuai, Z:: Global-stability problem for coupled system of differential equations on networks. J. Differ. Equ. 248 $1-20(2010)$

24. Gaines, R.E., Mawhin, J.L.: Coincidence Degree, and Nonlinear Differential Equations. Lecture Notes in Mathematics, vol. 568. Springer, Berlin (1977)

25. West, D.: Introduction to Graph Theory. Prentice Hall, Upper Saddle River (1996)

26. Hu, J., Zeng, C.N., Tan, J.: Boundedness and periodicity for linear threshold discrete-time quaternion-valued neural networks. Neurocomputing 267, 417-425 (2017)

27. Lu, S.P., Guo, Y.Z., Chen, L.J.: Periodic solutions for Lienard equation with an indefinite singularity. Nonlinear Anal., Real World Appl. 45, 542-556 (2019)

28. Lu, S.P., Jia, X.W.: Homoclinic solutions for a second-order singular differential equation. J. Fixed Point Theory Appl. 20 $101-115(2018)$

29. Yu, Y.C., Lu, S.P.: A multiplicity result for periodic solutions of Lienard equations with an attractive singularity. Appl. Math. Comput. 346, 183-192 (2019)

30. Du, B.: Stability analysis of periodic solution for a complex-valued neual networks with bounded and unbounded delays. Asian J. Control 20, 881-892 (2018)

31. Du, B., Lian, X., Cheng, X.: Partial differential equation modeling with Dirichlet boundary conditions on social networks. Bound. Value Probl. 2018, Article ID 50 (2018)

\section{Submit your manuscript to a SpringerOpen ${ }^{\circ}$ journal and benefit from:}

- Convenient online submission

- Rigorous peer review

- Open access: articles freely available online

- High visibility within the field

- Retaining the copyright to your article

Submit your next manuscript at $\gg$ springeropen.com 